\title{
Electrical classification of single red blood cell deformability in high- shear microchannel flows
}

\section{$\operatorname{AUTHOR}(S)$ :}

Katsumoto, Yoichi; Tatsumi, Kazuya; Doi, Tatsuki; Nakabe, Kazuyoshi

\section{CITATION:}

Katsumoto, Yoichi ...[et al]. Electrical classification of single red blood cell deformability in high-shear microchannel flows. International Journal of Heat and Fluid Flow 2010, 31(6): 985-995

\section{ISSUE DATE:}

2010-12

URL:

http://hdl.handle.net/2433/131864

\section{RIGHT:}

(C) 2010 Elsevier Inc:; This is not the published version. Please cite only the published version.; この論文は出版社版でありません。引用の際に は出版社版をご確認ご利用ください。 


\title{
Electrical Classification of Single Red Blood Cell Deformability in High-Shear Microchannel Flows
}

\author{
Yoichi Katsumoto $^{1}$, Kazuya Tatsumi ${ }^{1,2}$, Tatsuki Doi ${ }^{1}$ and Kazuyoshi Nakabe ${ }^{1,2}$ \\ 1. Department of Mechanical Engineering and Science, Kyoto University, Japan \\ 2. Advanced Research Institute of Fluid Science and Engineering, Kyoto University
}

\begin{abstract}
A sensor that can efficiently and sequentially measure the deformability of individual red blood cell (RBC) flowing along a microchannel is described. Counter-electrode-type microsensors are attached to the channel bottom wall, and as RBCs pass between the electrodes, the time series of the electric resistance is measured. A RBC is deformed by the high shear flow to a degree dependent upon its elastic modulus. Hence, the profile of the resistance, which is unique to the shape of the RBC, can be analyzed to obtain the deformability of each cell. First, theoretical and experimental analyses were conducted to identify the specific AC frequency at which the effect of the electric double layer formed on the electrode surface is minimized. Measurements were then conducted upon samples of normal human RBCs and glutaraldehyde-treated (rigidified) RBCs to evaluate the feasibility of the present method. In addition, simultaneous visualization of RBC deformation was performed using a high-speed camera. Normal RBCs were observed to have a degree of deformation index (DI) of 0.5, whereas the rigidified RBCs was $D I=0$ in the microchannel. The experimental measurements showed a strong correlation between the half-width of the maximum of the resistance distribution and the DI of the RBC.
\end{abstract}

Key words: $\quad$ Red blood cell, Deformability, Microchannel, Electric sensor, Laminar flow, Medical engineering 


\section{Introduction}

The measurement of the deformability of a red blood cell (RBC) is an important issue not just to clarify the hydrodynamic characteristics of the blood as fluid viscosity (Sugihara-Seki, 2005) and electric permeability (Katsumoto et al., 2008), but also to diagnose the initial symptoms of diseases during clinical investigation. For example, Plasmodium falciparum, a highly infectious parasite that causes severe anemia in a number of tissues and organs (Miller et al., 2002), significantly reduces the deformability of the RBCs by producing cytoadherence-related neoantigens that increase the internal viscosity and rigidity of the cytomembrane, even in the early "ring” stage (Dondorp et al., 2000; Suresh et al., 2005). Therefore, the development of a technique that can evaluate the deformability of RBCs through a cheap, fast, simple and accurate procedure would make a major contribution to the field of medicine, particularly in the area of clinical testing (Dondorp et al., 2000; Lim et al., 2006).

There are currently several means of measurement that are typically used to evaluate RBC deformability. One employs a viscometer to evaluate the deformability of bulk RBC solutions by considering fluid viscosity (Chien, 1975). Deformability can also be measured visually by using a rheometer to measure the extension rate of RBCs subjected to high shear stress that is generated in a coaxial rotating cylinder (Dobbe et al., 2002). These types of measurements can be termed bulk measurements since it is the bulk properties of the fluid that are often measured. On the other hand, there are methods that evaluate the deformability of each single cell. Optical or laser tweezers (Hénon et al., 1999; Suresh et al., 2005; Shelby et al., 2003) stretch a single RBC by applying force to the beads attached to the cell membrane, then evaluating the extension rate. The micro glass pipette (Engström et al., 1992; Mohandas and Evans, 1994), which is a simple yet effective traditional instrument, measures the membrane tension by visually analyzing the deformation of a RBC to which a certain aspiration pressure is applied in a micro glass pipette. There are other new methods as well, that use electric force to tense the 
RBC (Engelhardt and Sackmann, 1988), or that measure the unsteady behavior of RBCs in shear flows (Liu et al., 2007). Bulk measurements, however, tend to present a problem in terms of measuring resolution, especially when the number density of infected RBCs is small. This is not desirable for the clinical testing for diseases that are still in the early stages. Measuring schemes that evaluate the deformability of each single RBC, on the other hand, offer much greater accuracy, even though the number of available sample cells to be tested is limited.

The motivation for the present study is to develop a microsensor that can measure the deformability of a single RBC sequentially, by using hydrodynamic force and electrical sensors applied in a microchannel. Recently, Korin et al. (2007) visualized the behavior of RBCs in a microchannel and measured their cell deformation by analyzing the images of the cells as they were stretched by the high shear flow. The use of a microchannel offers the advantages of reduced cost, measuring time, and sample volume. Their method, however, requires high resolution image-recording equipment, which can lead to increased cost and the need for equipment that is far less compact. In contrast, the sensor proposed in the present study is based on using electrical means of measurement to evaluate RBC deformation. Specifically, platinum micro membrane electrodes are attached to the channel bottom wall, and a time series of the electric resistance is measured using an $\mathrm{AC} \mathrm{I}-\mathrm{V}$ method as the $\mathrm{RBC}$ passes between the electrodes. Compared with the previously mentioned means of optical measurement, this method can markedly decrease the size, cost and measuring time of the device used. Another asset is its greater compatibility with existing RBC counting equipment.

This paper reports upon an experiment that was carried out to evaluate the feasibility of the present sensor. A microchannel with micro membrane electrodes attached to the bottom wall was fabricated, so that when the RBC passes the electrodes, the distribution of the electric resistance could be measured and analyzed. To evaluate the relationship between the resistance profile and the deformability of the RBCs tested, two types of samples were used: normal 
human RBCs and glutaraldehyde-treated rigidified cells. In addition to this, visualization using a high-speed camera was performed simultaneously with the resistance measurements, to measure the location and shape of the RBC when it passes the electrodes. In order to validate the present sensor, these results were then compared with those obtained by electrical measurement.

\section{Nomenclature}

$a: \quad$ major axis of the RBC (= streamwise length of the cell) [m]

$b: \quad$ minor axis of the RBC (= spanwise length of the cell) [m]

Cv: $\quad$ coefficient of variance

$d_{\mathrm{RBC}}: \quad$ diameter of the RBC modeled as a sphere [m]

$D I: \quad$ deformation index $(=(a-b) /(a+b))$

$f: \quad$ AC frequency applied to the electrodes $[\mathrm{Hz}]$

$H_{1}$ : channel height at the location of electrodes and central inlet [m]

$\mathrm{H}_{2}$ : channel height [m]

$l_{\mathrm{s}}: \quad$ width of the sensor electrode [m]

$l_{\mathrm{g}}: \quad$ width of the guard electrodes [m]

$l_{s g}: \quad$ gap width between the sensor and guard electrodes [m]

$m: \quad$ average value

$R, R\left(x_{\mathrm{RBC}}\right): \quad$ resistance between sensor and ground electrodes [ $\left.\Omega\right]$

$\Delta R_{\mathrm{x}}: \quad$ resistance difference at $x_{\mathrm{RBC}}\left(=R\left(x_{\mathrm{RBC}}\right)-R(\infty)\right)[\Omega]$

$\Delta R_{0}: \quad$ maximum resistance difference $(=R(0)-R(\infty))[\Omega]$

$R_{\mathrm{s}}: \quad$ shunt resistance $[\Omega]$

Re: $\quad$ Reynolds number

$t: \quad$ time $[\mathrm{s}]$ 
$t_{\mathrm{cm}}: \quad$ membrane thickness $[\mathrm{m}]$

$V_{1}: \quad$ electric potential at upper port [V]

$V_{2}$ : electric potential of sensor electrode [V]

$w_{\mathrm{s}}: \quad$ distance between the sensor and ground electrodes $[\mathrm{m}]$

$W: \quad$ channel width $[\mathrm{m}]$

$x, y, z$ : Cartesian coordinates [m]

$x_{\mathrm{RBC}}, y_{\mathrm{RBC}}, z_{\mathrm{RBC}}$ : streamwise, spanwise and height positions of $\mathrm{RBC}$ when crossing the electrodes [m]

$Z^{*}: \quad$ impedance $[\Omega]$

$\delta: \quad$ half bandwidth [m]

$\varepsilon: \quad$ electric permittivity

$\varepsilon^{*}: \quad$ complex electric permittivity

$\varepsilon_{0}: \quad$ vacuum electric permittivity

$\kappa: \quad$ electric conductivity $[\mathrm{S} / \mathrm{m}]$

$v: \quad$ coefficient used in calculating the complex permittivity of RBC

$\omega: \quad$ angular frequency $[\mathrm{rad} / \mathrm{s}]$

$\sigma: \quad$ standard deviation

$\phi$ : $\quad$ volume ratio

subscripts

cp: $\quad$ value of cytoplasm

cm: value of cytomembrane

a: $\quad$ value of solution

RBC: value of RBC

sus: $\quad$ value of RBC suspended in solution 


\section{Measurement Physics}

Figure 1 shows a schematic diagram of the measuring method employed in this study. A pair of platinum membrane electrodes was attached to the bottom wall of a microchannel, and the time series of the electric resistance between them was measured. The electric conductivity of the red blood cell (RBC) cytomembrane is less than $1 \times 10^{-6} \mathrm{~S} / \mathrm{m}$, a value small enough for the cytomembrane to be considered an insulating material, as compared with the cytoplasm and normal saline solution. Therefore, the resistance between the electrodes increases significantly when RBCs pass between them in the microchannel flow due to the cytomembrane's strong electric insulating property. As the RBCs flow in the channel, they are stretched and deformed by the high shear stress provided by the steep velocity gradient of the flow. As mentioned above, the current distribution is interfered with mainly by the cytomembrane when a low-frequency AC electric field (below $\sim 10^{6} \mathrm{~Hz}$ ) is applied. In this case, the current density distribution will be affected by the shape of the cytomembrane; in other words, the electric resistance measured by the electrodes is dependent on the cell shape.

The time series profile of the measured resistance, e.g., maximum peak or the half-width of the maximum, is affected not only by the shape of the RBC, but also by its size and position within the flow, and the fluid properties. The effect of the fluid properties can be eliminated by normalizing the measured resistance, as will be discussed in Section 4.2. In this case, if the position of the RBC passing the sensor electrodes can be precisely controlled, then the resistance will depend principally on the shape of the RBC. Since the shape of the RBC indicates the deformation of the cell under the constant shear stress conditions, it can be used to calculate the deformability of the RBC. Thus, the deformability of the RBC can be evaluated by analyzing the profile of the time series of the resistance as the RBC passes the electrodes. 
Figure 2 (a) shows the equivalent circuit of a single RBC that is suspended in a solution and located between the electrodes. In this case, the circuit between the electrodes consists of cytoplasm, cytomembrane, the solution, and the electric double layer formed on the electrode surface. In order to present the time-dependent change of the electric characteristics when the RBC passes between the electrodes, this circuit can also be simplified as a parallel component of variable resistance and capacitance connected in series with the component of the electric double layer, as shown in Fig. 2 (b).

As can be seen, the accuracy of the measurement largely relies upon the degree of difference between the resistances of the buffer solution and the RBC membrane. If a solution of high ionic concentration, such as isotonic sodium chloride solution, is used, however, the electric double layer formed on the electrode surface will degrade the sensitivity of the sensor. That is, the electric charge at the capacitance of the double layer leads to a potential drop and an increase in the apparent resistance of the solution. This problem can be solved by applying a high frequency AC electric field to the electrodes to reduce the impedance of the electric double layer. In contrast, since the cell membrane consists of insulating resistance and capacitance, too great an increase in the frequency of the electric field can incur a decrease in cell resistance, as well. In defining the frequency range to be applied on the electrodes, we therefore find ourselves facing a trade-off between different problems. These upper and lower limits of the frequency were evaluated analytically and experimentally as described in Section 3.3, where the appropriate value for the present measurement is defined.

\section{Experimental Setup}

\subsection{The microchannel and electrodes}

Figure 3 shows a schematic diagram of the channel and the sensor electrodes used in this study. The channel width, $W$, is $1 \mathrm{~mm}$, which is much larger than the channel heights $H_{1}$ and $H_{2}$. 
The channel flow can therefore be considered a two-dimensional flow, and the RBC is deformed by receiving the shear stress caused by the velocity gradient generated in the height direction.

As shown in Fig. 3 (b), the channel has three inlets. An RBC suspended in phosphate buffered saline (PBS; Amresco) is supplied from the central inlet while PBS solution only, is supplied from the side inlets. Due to the low Reynolds number of the flow in the microchannel, the flow is in laminar regime, and there is just a small degree of spreading of the flow supplied from the central inlet. By changing the flow rate of each side flow, therefore, the spanwise position of the RBC can be controlled so that it will pass between the electrodes.

When an RBC is placed in a channel shear flow, the cell receives a lift force that is attributed to the so-called Fåhraeus effect (Goldsmith et al., 1989). This lift force moves the RBC away from the channel wall on which the sensor electrodes are embedded, which apparently leads to a decrease in both the measuring resistance and the accuracy of the sensor. To tackle this problem, the channel height at the central inlet and sensor part, $H_{1}$, was made smaller than that in other areas, $H_{2}$. As can be seen in Fig. 3 (a), a backward-facing step was placed at the point where the central inlet and the main channel merge, and a forward-facing step was placed immediately upstream of the electrodes. The RBC supplied from the central inlet would thus remain in a lower position in the main channel, and the downwash flow at the forward-facing step would carry the RBC downward, closer to the bottom wall of the channel.

At the forward-facing step, the strong downward flow will not only guide the RBC toward the channel bottom wall, but also produce an elongational flow that will deform the RBC in addition to the effect of the shear stress. However, the flow Reynolds number in the present study is very small, $4.8 \times 10^{-4}$, that requires a short streamwise length for the flow to redevelop. Since the distance between the forward-facing step and the electrode is $60 \mu \mathrm{m} \approx 6 \mathrm{H}_{2}$, the effect of this enlongational flow is considered to be negligible in the area near the electrodes in this study. Yet, it should be noted that the distance between the forward-facing step and the electrodes 
should be considered carefully in order to eliminate such an elongational flow effect on the cell deformation.

The combined effects of these structures enabled us to control the RBC height position, $z_{\mathrm{RBC}}$, so that it would be close to the electrodes. Since the ratio of $H_{1}$ to $H_{2}$ is 5.2, the height position of the RBC will be less than $1.9 \mu \mathrm{m}$ when it passes the electrodes. This was confirmed by the visualization measurement that $z_{\mathrm{RBC}}$ was less than $3 \mu \mathrm{m}$ during the experiment.

Counter-type platinum membrane electrodes were transversely attached to the bottom wall of the channel, as shown in Fig. 3 (c). Guard electrodes were placed on both sides of the sensor electrode. An electrical potential identical to that applied to the sensor electrode was supplied to these guard electrodes in order to reduce the fringe effect of the electric field observed at the edges of the sensor electrode. This leads to an increase of the sensitivity in the sensor.

\subsection{Fabrication process of the microchannel and electrodes}

The microchannel illustrated in Fig. 3 was made of PDMS (poly-dimethylsiloxane) and was fabricated by using SU-8 (MicroChem Co.) as a casting mold. This SU-8 mold was fabricated through an lithography process. First, an optical mask was prepared by using film projection equipment (Fujifilm Co. Enlarger FD690) to print the channel pattern on an emulsion photo plate (Konica Minolta Inc. high precision plate SN-2). An SU-8 layer was spin-coated on a glass slide using a spin coater (Mikasa Co. Ltd.; 1H-D7), and was then exposed to UV light through the optical mask by using a mask aligner (Nanometric Technology Inc.: LA310), with which the channel pattern was printed on the layer. After post-baking with a hot plate, and an etching process performed with the developer (MicroChem Co.; Su-8 Developer), an SU-8 mold was created. PDMS (Shin-Etsu Chemical Co. Ltd.; KE-106) mixed with a hardening agent (Shin-Etsu Chemical Co. Ltd.; CAT-RG) in liquid phase was then poured over the SU-8 mold, and was hardened by baking with a hot plate for 6 hours at $80^{\circ} \mathrm{C}$. The PDMS channel was then 
removed and attached to a glass slide, on which the platinum membrane electrodes were printed.

The platinum electrodes were fabricated through sputtering and lift-off processes using a sputter device (ULVAC KIKO Inc: SCOTT-C3) and photo resist (MicroChem Co.; ZPN). In this case, the photo mask was first prepared using mask blanks (ULVAC COATING Co.; DUFR-2506(P)-L) and the Electron Beam Lithography system. The lift-off resist layer of ZPN with the patterns of the electrodes was then formed on a glass slide using the spin coater, mask aligner, hot plate, and developer (ZEON Co.; ZTMA100). The platinum was then sputtered on this resist-patterned glass slide. It should be noted that in order to obtain a stronger contact between the platinum and glass, a contact layer of chrome was sputtered on the glass slide before the platinum layer was formed. The thickness of these layers was on the order of hundreds of nm, as measured by a surface profiler. The ZPN resist was removed using acetone, and the metal membrane with the pattern of the electrodes was then formed on the glass surface. Finally, the PDMS channel and the glass slide with platinum electrodes were bonded together.

In order to increase the microscopic surface area of the electrodes to reduce the impedance of the electric double layer formed on them when the voltage is supplied, the electrodes were plated with platinum nanoparticles. This plating process took place as follows. First, the electrode was washed by filling the channel with $0.5 \mathrm{M}$ sulfuric acid for $10 \mathrm{~min}$. The channel was then washed by running pure water through it. The channel was then filled with a solution of $30 \mathrm{mg} / \mathrm{mL}$ hexachloroplatinic $\left(\mathrm{H}_{2} \mathrm{PtCl}_{6}\right)$ and $0.3 \mathrm{mg} / \mathrm{mL}$ lead acetate $\left(\mathrm{CH}_{3} \mathrm{COO}\right)_{2} \mathrm{~Pb}$. 0.5V DC voltage was then applied to the electrode, and the platinum nanoparticles were plated onto the surface. The channel and electrode were then washed with pure water. After filling with $0.5 \mathrm{M}$ sulfuric acid for $10 \mathrm{~min}$, the channel was again washed with pure water.

\subsection{Setup of the electrical measurement}

The circuit used in this study to measure the resistance between the electrodes is shown in 
Fig. 4. The shunt resistor of $R_{\mathrm{s}}=20 \mathrm{k} \Omega$ for current detection was connected serially to the sensor electrode of which the opposite side is grounded. A sinusoidal wave of AC voltage with an amplitude of $1 \mathrm{~V}$ and a frequency of $10 \mathrm{kHz}$ was applied to the upper part of the shunt resistor using a function generator (NF Co.; WF-1973). The guard electrodes were connected to the sensor electrode through a JFET input Op-Amp (National Semiconductor Co.; LF411), and the potential of these electrodes were kept identical to that applied to the sensor electrode. The upper port potential $V_{1}$ and the sensor electrode potential $V_{2}$ were impedance-converted by the Op-Amp (Analog Devices Inc.; AD621). These signals were recorded by a computer through an A/D board (National Instruments Co.; PCIe-6251) with a sampling frequency of $600 \mathrm{kHz}$. The complex impedance between the sensor and ground electrodes in this circuit can be obtained as Eq. (1). The resistance between the electrodes, $R$, was obtained from $Z^{*}$ after measurement.

$$
Z^{*}(\omega)=R_{s} \frac{V_{2}^{*}}{V_{1}^{*}-V_{2}^{*}}
$$

As was mentioned in Section 2, the AC electric field needed to be applied at a specific frequency in order to reduce the impedance of the electric double layer formed on the electrodes, and without decreasing the resistance of the RBC. Before carrying out the measurement using RBCs, the impedance characteristic of the electrodes was evaluated analytically and experimentally in order to define the specific AC frequency to be applied to the electrodes.

The maximum limit of the frequency to be applied can be estimated analytically as follows. First, the complex permittivity, $\varepsilon^{*}$, can be defined as Eq. (2) using the electric permittivity, $\varepsilon$, and conductivity, $\kappa$.

$$
\varepsilon^{*}=\varepsilon-i \frac{\kappa}{\varepsilon_{0} \omega}
$$

When a RBC is modeled as a sphere with diameter $d_{\mathrm{RBC}}$ and membrane thickness $t_{\mathrm{cm}}$, as 
shown in Fig. 5, the equivalent complex permittivity, $\varepsilon_{R B C}^{*}$, can be written as Eq. (3) (Hanai, 1968).

$$
\varepsilon_{R B C}^{*}=\varepsilon_{c m}^{*} \frac{2(1-v) \varepsilon_{c m}^{*}+(1+2 v) \varepsilon_{c p}^{*}}{(2+v) \varepsilon_{c m}^{*}+(1-v) \varepsilon_{c p}^{*}}
$$

$v=\frac{d_{R B C}-2 t_{c m}}{d_{R B C}}$

$\varepsilon_{c p}, \kappa_{c p}, \varepsilon_{c m}$ and $\kappa_{c m}$ are the relative permittivity and conductivity of the cytoplasm and cytomembrane, respectively.

If the RBC is suspended in a solution of relative permittivity, $\varepsilon_{a}$, and conductivity, $\kappa_{a}$, with a volume ratio of $\phi$, the overall relative complex permittivity, $\varepsilon_{\text {sus }}^{*}$, can be analytically derived from Eq. (4).

$$
\varepsilon_{\text {sus }}^{*}=\varepsilon_{d}^{*} \frac{2(1-\phi) \varepsilon_{a}^{*}+(1+2 \phi) \varepsilon_{R B C}^{*}}{(2+\phi) \varepsilon_{a}^{*}+(1-\phi) \varepsilon_{R B C}^{*}}
$$

Figure 6 shows the effects of the frequency, $f$, on the permittivity, $\varepsilon_{\text {sus }}$, and conductivity, $\kappa_{\text {sus }}$, obtained from Eqs. (3) and (4) in the case of a RBC suspended in phosphate buffered saline (PBS) with a volume ratio of $\phi=0.05$. The values used in the equations are $\varepsilon_{c p}=50$, $\kappa_{c p}=0.67 \mathrm{~S} / \mathrm{m}, \varepsilon_{c m}=5, \kappa_{c m}=1 \times 10^{-7} \mathrm{~S} / \mathrm{m}, t_{c m}=5 \mathrm{~nm}, d_{\mathrm{RBC}}=6 \mu \mathrm{m}, \varepsilon_{a}=78.3$, and $\kappa_{a}$ $=1.67 \mathrm{~S} / \mathrm{m}$, respectively.

In Fig. $6, \kappa_{\text {sus }}$ increases with $f$ in the area above several $\mathrm{MHz}$, which is designated in the figure by a vertical line. This shows the dielectric relaxation behavior of the cell and the solution. This indicates that the cytomembrane acts as an insulator when the frequency is low, while the cytoplasm is directly measured when the frequency is high. As mentioned above, the present sensor exhibits higher performance when there is a larger difference between the resistance of the solution and the RBC. For this reason, a frequency that is smaller than this 
threshold value should be used during the measurement.

On the other hand, the lower limit of the frequency was experimentally obtained by first filling the sensor channel with PBS solution, then measuring the electric resistance between the electrodes with an impedance analyzer (Agilent: 4294A).

Figure 7 shows how the frequency of the applied AC voltage, $f$, affected the resistance, $R$. The white and black circles in the figure present the results when the platinum bare electrodes and platinum black-plated electrodes mentioned in Section 3.2 were used, respectively. In the figure, the resistance $R$ decreases as $f$ increases, and becomes constant when each of the two were used. This supports our observation that when $f$ is not sufficiently high, an electric double layer is formed on the electrode surfaces, which increases the apparent resistance measured by the electrodes.

Comparing the results of the bare electrodes with those of the platinum black-plated ones, one can see that the frequency range at which $R$ begins to increase is located in the lower frequency side in the case of the plated electrodes. This indicates a reduction of the electric double layer effect. As mentioned in Section 3.2, this can be attributed to the increase in the surface area caused by the porous media-like layers of platinum nanoparticles generated on the surface of the electrodes. In addition to this change in the frequency characteristics, it should be noted that the resistance in the case of the platinum black-plated electrodes was smaller than in the case of the bare electrodes. This can be attributed to the fact that the plating is uniformly distributed on the surface, leading to a decrease in the distance between the electrodes.

Based upon these results, an AC frequency $f$ of $10 \mathrm{kHz}$ was employed in this study.

\subsection{Setup of the visualization measurement}

Along with the above electrical measurements, visualization measurement was simultaneously carried out in order to verify the shape and position of the RBCs when they 
passed the forward-facing step and the electrodes. The experimental apparatus employed is shown in Fig. 8. The measurement was carried out using an invert-type microscope (Olympus Co.; IX-71) with a halogen lamp as the light source. The objective lens was a long working distance lens with $\times 100$ magnification and $N A=0.8$ (Olympus Co.; LMPLFLN100X). The images were recorded by a high speed camera (Vision Research; Phantom V7.3). The frame rate of the camera was 1000 frames/s and the CCD resolution was $800 \times 650$ pixels. The optical resolution of the image in combination with the lens was $0.073 \mu \mathrm{m} / \mathrm{pixel}$. The focusing depth of the image was $1.7 \mu \mathrm{m}$.

\subsection{Preparation of normal and rigidified RBCs}

Sample blood was collected from healthy human volunteers. To prepare the normal RBCs, the blood was first washed two times via the following procedure: blood was suspended in PBS, centrifuged for $10 \mathrm{~min}$ at $2000 \mathrm{~g}$, and the supernatant was removed. The precipitated RBCs were collected and suspended in PBS with 8 wt\% polyvinylpyrrolidone (PVP).

Rigidified RBCs were prepared by treating normal RBCs with glutaraldehyde. In this case, the glutaraldehyde (Nakalai tesque Co.) was first diluted with PBS by $0.025 \mathrm{wt} \%$. These RBCs were washed twice in the same way as the normal RBCs, then the precipitated RBCs were suspended in the glutaraldehyde-PBS solution. After settling for $30 \mathrm{~min}$ at room temperature, the RBCs were washed twice. The collected RBCs were then suspended in a PBS solution with 8 wt\% PVP. The measurements were carried out within 3 hours after the preparation of these RBCs.

\subsection{Flow conditions}

Korin et al. (2007) reported that the shear stress required to deform a RBC with a deformation index DI of 0.5 is approximately $15 \mathrm{~Pa}$. When water is used as the working fluid, it 
is difficult to obtain this shear stress by increasing the flow rate, given the height of the present channel, due to the limitation of the maximum frame rate of the high-speed camera. Furthermore, increasing the velocity of the RBC leads to a decrease in the measuring time between the sensor electrodes. However, since the sampling ratio of the A/D board is limited, a greater velocity will only reduce the accuracy of the resulting resistance profile. For this reason, PVP $\left(\right.$ MW $=3.6 \times 10^{5}$, Nacalai tesque; K-90) was mixed with the PBS. The viscosity of the solution as measured by a rheometer yielded a value of 0.08-0.09 Pa.s, which is approximately 100 times larger than that of the PBS solution alone. A shear stress sufficient to deform the RBC was thus achieved, even under the condition of a lower flow velocity.

These working fluids with and without RBCs were supplied through Teflon tubes to the central inlets and side inlets, respectively, by means of syringe pumps (Harvard Apparatus Co., econoflo; Nihon Kohden Co., CFV-3200). The mass flow rate of the flow supplied to the central inlet was $0.02 \mu \mathrm{l} / \mathrm{min}$. The mass flow rates of the side flows were basically $1.0 \mu \mathrm{l} / \mathrm{min}$, but were changed to the range of $\pm 0.2 \mu \mathrm{l} / \mathrm{min}$ in order to control the spanwise position of the flowing RBCs. In this case, the Reynolds number based on the hydraulic diameter of the cross-sectional area at the sensor, and the spanwise averaged velocity, was then $R e=4.8 \times 10^{-4}$.

\section{Results and Discussion}

\subsection{Results of visualization measurement}

Figure 9 presents a photo taken from below the electrodes and the forward-facing step. The flow direction is from left to right, and the vertical black line is the forward-facing step. The origin is centered between the sensor electrode and the ground electrode. The edges of the electrodes are relatively rough, due to the platinum particles plated onto their surface.

Figure 10 shows photos presenting the behavior of a normal RBC as it passes the forward-facing step and the electrodes. Since the raw image of the RBC is difficult to distinguish 
from the background, the outline of the cell is depicted by a white line. The time described in the images is the time relative to $t=0 \mathrm{~s}$, which is the moment when the location of the RBC is $x_{\mathrm{RBC}}=0$.

In the figure, the RBC is deformed and stretched in the streamwise direction in response to the two-dimensional shear flow generated in the channel. The RBC deformed most when passing the forward-facing step, as shown in the photo at $t=-0.020 \mathrm{~s}$. This is due to the downward flow generated at the forward-facing step, which not only increases the shear stress, but also carries the RBC toward the area close to the bottom wall where a large shear stress is formed. As the RBC flowed downstream, the deformation rate of the cell gradually decreased. This can be attributed to the Fåhraeus effect (Goldsmith et al., 1989), which in this case results as follows: Due to the shear stress, a lift force impacts the RBC, causing it to move toward the center of the channel, which results in reduction of the shear stress and deformation of the RBC.

One can see that the spanwise position of the RBC does not change as it flows downstream. Therefore, the spanwise position can be effectively controlled by the side flows so that the RBC will pass the center of the electrodes. It should be noted, however, that there is some variation in the spanwise position of each cell on the order of $\pm 3 \mu \mathrm{m}$. This is due to the fact that the width of the central inlet is $50 \mu \mathrm{m}$, which is much larger than the distance between the electrodes, which is $w_{\mathrm{s}}=$ $5.2 \mu \mathrm{m}$. The influence that this variation in the RBC spanwise position has on the electrical measurements will be discussed in Section 4.2.

Figure 11 presents photos of a glutaraldehyde-treated rigidified RBC passing the forward-facing step and the electrodes. In contrast to a normal RBC, the rigidified RBC maintains its biconcave surface, and exhibits no observable deformation. The RBC rotates as it flows, in what is called a 'flipping' motion. When a normal RBC is suspended in a shear flow, the cytomembrane not only deforms, but it also rotates together with the cytoplasm, causing a flow circulation inside the cell. This is called the 'tank treading motion' of the cell. The torque caused by the shear flow is converted into this motion of the membrane, which prevents the cell from flipping (Olla, 1997). 
However, since the cytomembrane of the glutaraldehyde-treated RBC is highly rigidified in order to maintain its biconcave shape, the tank treading motion cannot be activated. The cell therefore flips as would any solid object that is immersed in a shear flow.

It is obvious that if this RBC rotation takes place in the area between the electrodes, the resistance of RBC with different orientation will be measured. During the measurement, however, the RBC was oriented so as to have the biconcave surface aligned with the z-direction at the forward-facing step. Most of the RBC rotated at the downstream of the electrodes as shown in Fig. 11. This is considered to be attributed strong shear stress and the elongational flow generated at the step that has been previously discussed. This RBC rotation is a significant problem for practical use of the present sensor, and improvement of the sensor (e.g. changing the channel height or spanwise width at the sensor part) is considered as future work.

Table 2 shows the average value of the streamwise and spanwise lengths of the RBC, $a$ and $b$, and the $D I$ value obtained during the visualization. $D I$ presents the degree of deformation and is defined as follows:

$$
D I=\frac{a-b}{a+b}
$$

The number of samples and the standard deviation are also shown in the table.

In the case of a rigidified RBC, the average values of $a$ and $b$ are identical and DI becomes 0 , which indicates that the RBC is not deformed and has a circle-projected shape. On the other hand, in the case of a normal RBC, since the cell is stretched in the streamwise direction, as shown in Fig. $10, a$ becomes larger than $b$, and $D I$ is 0.57 . As can be seen in the standard deviation, the $D I$ varies for some degree in the cases of normal and rigidified RBCs. This variation is considered to be attributed to the difference in the original deformability of the RBC, and to the influence of the variation in the height position of the RBC when passing between the electrodes, $z_{\mathrm{RBC}}$. Even among the healthy RBCs, a difference in the membrane or cytoplasm characteristics still exists (e.g. owing the aging effects. Shiga et al. 1985). This leads to the difference in the deformability. On the other 
hand, as previously described, the $\mathrm{RBC}$ height position $z_{\mathrm{RBC}}$ varies in the present sensor. This will incur a difference of the shear stress working on the RBC that leads to a variation in DI. The effect of this variation will also be discussed in Section 4.2.

\subsection{Results of electrical measurement}

Figure 12 shows the electrical resistance, $\Delta R_{\mathrm{x}} / \Delta R_{0}$ distribution, in relation to the streamwise position of the RBC, $x_{\mathrm{RBC}}$. The original resistance distribution measured by the circuit and $\mathrm{A} / \mathrm{D}$ board is obtained in a time sequential form. Therefore, $x_{\mathrm{RBC}}$ is obtained by multiplying this time line by the streamwise velocity of each $\mathrm{RBC}$, as estimated from the previously mentioned visualization results. $\Delta R_{\mathrm{x}}$ is the resistance difference obtained by subtracting the base line resistance, which is the value when the RBC is located sufficiently far from the electrodes $R(\infty)$, from the original readout $R\left(x_{\mathrm{RBC}}\right) . \Delta R_{0}$ is the resistance difference when $x_{\mathrm{RBC}}=0$, i.e. $R(0)-R(\infty)$. In other words, this is the value when $\Delta R_{\mathrm{x}}$ reaches its maximum.

The results shown in Fig. 12 are typical for normal $(D I=0.57)$ and rigidified $(D I=0.0)$ RBCs, and correspond to the values for RBCs shown in Figs. 10 and 11, respectively. In the figure, a broader distribution is observed in the case of a normal RBC than for rigidified one. This indicates the possibility of using the half-width of the maximum of the $\Delta R_{\mathrm{x}} / \Delta R_{0}$ distribution, $\delta$, in classifying the normal and rigidified RBCs.

As described in Section 4.1, the spanwise position of the RBCs varies when passing the sensor electrode. This is attributed to the width of the central inlet of the channel, which is larger than the distance between the sensor and ground electrodes. This variation can affect the $\Delta R_{\mathrm{x}} / \Delta R_{0}$ distributions. Therefore, the influence of the spanwise position of the RBC on $\Delta R_{0}$ and $\delta$ must be evaluated first, before addressing the relationship between $\Delta R_{0}$ and $\delta$ and the deformation index, $D I$.

Figure 13 show a scattering diagram of $\Delta R_{0}$ and $\delta$ for the normal and rigidified RBCs. The 
abscissa $y_{\mathrm{RBC}}$ is the spanwise position of the RBC at the instant when its streamwise location is $x_{\mathrm{RBC}}=0$. The area at both ends of the graph parted by the vertical dash lines shows the location of the sensor and ground electrodes. The solid and dashed lines in the figure present the least square approximation taken for the normal and rigidified RBCs, respectively.

In Fig. 13 (a), $\Delta R_{0}$ decreases linearly as the RBC position $y_{R B C}$ becomes closer to the ground electrode. On the other hand, $\delta$ increases as $y_{R B C}$ decreases, as shown in Fig. 13 (b). This can be attributed to the following dynamic. As seen in Figs. 3 and 9, there are three electrodes at the sensor side - the sensor and two guard electrodes - while the ground side has just one. Due to this asymmetric shape, the effect of the guard electrodes reducing the fringe components of the current flux becomes stronger on the sensor side, and diminishes in the area closer to the ground side. In other words, the current flux distribution spreads widely in the streamwise direction as it gets closer to the ground electrode, which leads to a decrease in current density in the area closer to the ground electrode. Since the resistance will increase largely when the RBC obstructs a large amount of current fluxes, i.e. an area with larger current density, $\Delta R_{0}$ increases when the RBC passes between the electrodes in a location closer to the sensor side. On the other hand, since the current density shows a wider and moderate distribution at the location close to the ground electrode, $\Delta R_{\mathrm{x}}$ begins to increase at a streamwise location distant from $x=0$. In combination with the decrease in $\Delta R_{0}$, therefore, the half-width of the maximum of $\Delta R_{\mathrm{X}} / \Delta R_{0}, \delta$ increases in the area closer to the ground electrode, as observed in Fig. 13 (b).

As observed in Fig. 13, the spanwise position of the RBC, $y_{R B C}$, appears to affect $\Delta R_{0}$ and $\delta$ linearly. Therefore, the linear component shown in the L.S.M. line in the figure, is subtracted from each value in order to remove the effect of the asymmetric distribution of the current density, as mentioned above. The results are shown in Fig. 14 in relation with the deformation index, DI, which is obtained from the visualization measurements. Since only two kinds of RBCs, normal and glutaraldehyde-treated rigidified ones, were measured in the present experiment, two large groups 
are found in the area near $D I=0.0$ and 0.5 .

In Fig. 14 (a), $\Delta R_{0}$ of the normal and rigidified RBCs overlap with each other and are of nearly equal value. Therefore, the difference in shape, i.e. difference in the deformability of the cell, cannot be distinguished by this value. In contrast, $\delta$ is larger for $D I=0.5$ as compared with $D I=0.0$, as shown in Fig. 14 (b). This is consistent with our discussion of the $\Delta R_{\mathrm{X}} / \Delta R_{0}$ distribution shown in Fig. 12. This therefore indicates that $\delta$ is an effective index for the classification of RBC deformability.

Another important factor in evaluating the effectiveness of the index in the classification of RBC deformability, can be the magnitude of error in the obtained values. In order to address this matter, RBCs with different DI should be measured, and an uncertainty analysis for the random error observed in the measurement should be performed. However, it would be difficult to prepare RBCs with gradually changing cell deformability, since glutaraldehyde treatment is usually used to fix the shape of the cells. Instead, a coefficient of variance defined as Eq. (6) will be discussed here to provide some information regarding the errors of the two indexes.

$$
C v=\frac{\sigma}{m} \times 100
$$

$\sigma$ and $m$ are the standard deviation and average values of the samples. The results are shown in Table 3.

In that table, $C v$ of $\Delta R_{0}$ is relatively large for the normal and rigidified RBCs compared with those in the case of $\delta$, indicating that the dispersion of the samples in $\Delta R_{0}$ is large for both types of RBCs. This shows that $\delta$ is a more reasonable index to evaluate the deformability of a RBC, as compared with $\Delta R_{0}$, not only because the value shows a larger variation with $D I$, but because it also shows a smaller dispersion, which might lead to a smaller degree of error.

$\delta$ shows a better performance than $\Delta R_{0}$ as an index to distinguish RBC deformability, a fact that can be attributed to the three-dimensional distribution of the current density formed in the area between the electrodes. When considering a current density distribution between two-point 
electrical charges as an extreme condition, the current density will attenuate by a square function in relation to the distance from the two-point charges. We expected to observe a similar distribution in the electrodes used in this study. That is, a high current density would be produced between the spanwise edges of the sensor and ground electrodes, and the current density would largely decrease with distance from the electrodes in the streamwise direction and particularly in the height direction. As mentioned in Section 2, the value $\Delta R$ is strongly related to the condition of how the RBC intercepts the current density distributions, i.e. the size of the cross-sectional area blocked by the cell, and the original current density in the area the cell is expected to pass through. If the RBC passes through an area with high current density, a larger $\Delta R$ would therefore be observed.

Considering, then, the current density pattern and the previously mentioned characteristics of $\Delta R, \Delta R_{0}$ is expected to be highly sensitive to the RBC height position at the moment it passes the electrodes, $z_{\mathrm{RBC}}$. In the present sensor, $z_{\mathrm{RBC}}$ is maintained as a constant due to the laminar flow in the microchannel, and the forward-facing step placed upstream of the electrodes. However, because the channel height of the central inlet $H_{1}$ is larger than the RBC diameter, there can be variations in $Z_{\mathrm{RBC}}$ on some level. This could explain why larger $C v$ is observed with $\Delta R_{0}$.

On the other hand, $\delta$ depends more on the pattern of the current density distribution in the streamwise direction than in the height direction. Therefore, if the $z_{\mathrm{RBC}}$ will not fluctuate in relation to the streamwise position of the RBC, $\delta$ can be expected to be less affected by the current density distribution in the height direction and the $z_{\mathrm{RBC}}$. Smaller $C v$ is therefore observed with $\delta$.

It should be noted here that a variation in the DI of normal and rigidified RBCs is observed in the figure. As described in Section 4.1, one of the reasons for this is considered to be due to the variation in the deformability of the $\mathrm{RBC}$ and $z_{\mathrm{RBC}}$. The correlation confirmed in the present study will be, therefore, the one between the deformation index DI and the half-bandwidth $\delta$ of the resistance distribution.

To address and explore these problems, further measurements and numerical simulation for the 
electric field and resistance characteristics need to be carried out. Furthermore, measurements of the exact deformability of the sample RBC using other equipments will be of significant and practical interest in order to evaluate the relation between the deformability and $\delta$.

In any case, it can be concluded that the feasibility of using microchannels and micro electric sensors to measure RBC deformability is validated, and the possibility of using $\delta$ as the index to evaluate the deformation rate of RBC has been affirmed.

\section{Conclusions}

A microsensor consisting of a microchannel and micro membrane-type electrodes was proposed and evaluated in order to measure the deformability of a single RBC by analyzing the resistance distribution observed when a RBC deformed by high shear flow passed between the electrodes. The feasibility of the sensor was experimentally examined using two types of samples: normal human RBCs and glutaraldehyde-treated rigidified human RBCs. The major results are as follows.

1. The frequency of the AC voltage to be applied to the sensor electrodes was determined analytically and experimentally. Specifying the appropriate frequency reduced the effects of the electric double layer formed on the electrodes without incurring the dielectric relaxation of the cell membrane. In addition to this, the electrodes were plated with platinum black in order to increase their surface area and reduce the effect of the electric double layer. This adjustment of the frequency, and the platinum plating, effectively increased the sensitivity of the sensor.

2. The backward- and forward-facing steps, which were located at the central inlet, and upstream of the electrodes, respectively, along with the side flows applied in the microchannel, were effective in controlling the height and spanwise position of the RBCs to induce them to pass close to the sensor electrodes.

3. In the visualization measurements, the deformation of a normal RBC to an ellipsoidal 
shape was observed, while the rigidified RBC maintained its biconcave shape. This result shows that the shear stress that was generated effectively controlled the deformation rate of the RBC by introducing a forward-facing step, along with the increase in fluid viscosity achieved by mixing polyvinylpyrrolidone into the solution.

4. A strong correlation was observed between the half-width of the maximum of the resistance distribution, $\delta$, and the deformation index of the RBC obtained from the visualization. In contrast, the correlation between the peak value of the resistance, $\Delta R_{0}$, and the deformation index, was weak. Furthermore, the variation of the samples was larger with $\Delta R_{0}$ than with $\delta$. These characteristics can be strongly attributed to the three-dimensional distribution of the current density formed between the electrodes. Consequently, $\delta$ performed positively for the classification of RBC deformability, and the feasibility of the present sensor was substantially verified.

\section{Acknowledgements}

This work was financially supported by the Ministry of Education, Culture, Sports and Science and Technology of Japan, and also partially by the CASIO Science Promotion Foundation.

\section{References}

Chien, S., 1975. Biophysical Behavior of Red Cells in Suspension, In: Surgenor, D. M. (Ed.) The Red Blood Cell, Academic Press, New York, 2, pp. 1031-1133.

Dobbe, J. G. G., Streekstra, G. J., Hardeman, M. R., Ince, C., Grimbergen, C. A., 2002. Measurement of the Distribution of Red Blood Cell Deformability Using an Automated Rheoscope, Cytometry, 50, 313-325. 
Dondorp, A. M., Kager, P. A., Vreeken, J., White, N. J., 2000. Abnormal Blood Flow and Red Blood Cell Deformability in Severe Malaria, Parasitology Today, 16, 228-232.

Engelhardt, H., Sackmann, E., 1988. On the Measurement of Shear Elastic Moduli and Viscosities of Erythrocyte Plasma Membranes by Transient Deformation in High Frequency Electric Fields, Biophysics J., 54, 495-508.

Engström, K., Möller, B., Meiselman H. J., 1992. Optical Evaluation of Red Blood Cell Geometry Using Micropipette Aspiration, Blood Cells, 18, 241-258.

Goldsmith, H. L., Cokelet, G. R., Gaehtgens, P., 1989. Robin Fåhraeus: Evolution of His Concepts in Cardiovascular Physiology, American J. Physiological Society, 257, 1005-1015.

Hanai, T., 1968. Electric Properties of Emulsions, In: Sherman, P. (Ed.), Emulsion Science, Academic Press, London, pp. 353-478.

Hénon, S., Lenormand, G., Richert, A., Gallet, F., 1999. A New Determination of the Shear Modulus of the Human Erythrocyte Membrane Using Optical Tweezers, Biophysical J., 76, $1145-1151$.

Katsumoto, Y., Hayashi, Y., Oshige, I., Omori, S., Kishii, N., Yasuda, A., Asami, K., 2008. Dielectric Cytometry with Three-dimensional Cellular Modeling, Biophysical J., 95 (6), 3043-3047.

Korin, N., Bransky, A., Dinnar, U., 2007. Theoretical Model and Experimental Study of Red Blood Cell (RBC) Deformation in Micro-channels, J. Biomechanics, 40, 2088-2095.

Lim, C. T., Zhou, E. H. and Quek, S. T., 2006. Mechanical Models for Living Cells - a Review, J. Biomechanics, 39, 195-216.

Liu, X., Tang, Z., Zeng, Z., Chen, X., Yao, W., Yan, Z., Shi, Y., Shan, H., Sun, D., He, D., Wen, Z., 2007. The Measurement of Shear Modulus and Membrane Surface Viscosity of RBC Membrane with Ektacytometry: A New Technique, Mathematical Biosciences, 209, 190-204. 
Miller, L. H., Baruch, D. I., Marsh, K., Doumbo, O. K., 2002. The Pathogenic Basis of Malaria, Nature, 415, 673-679.

Mohandas, N. and Evans, E., 1994. Mechanical Properties of the Red Blood Cell Membrane in Relation to Molecular Structure and Genetic Defects, Annual Reviews, 23, 787-818.

Olla, P., 1997. The Lift on a Tank-Treading Ellipsoidal Cell in a Shear Flow, J. Physics 2 France, 7, 1533-1540.

Shelby, J. P., White, J., Ganesan, K., Rathod, P. K., Chiu, D. T., 2003. A Microfluidic Model for Single-Cell Capillary Obstruction by Plasmodium Falciparum-infected Erythrocytes, PNAS, 100, $14618-14622$.

Shiga, T., Sekiya, M., Maeda, N., Kon, K., Okazaki, M., 1985. Cell Age-dependent Changes in Deformability and Calcium Accumulation of Human Erythrocytes, Biochimica et Biophysica Acta, 814, 289-299.

Sugihara-Seki, M. and Fu, B. M., 2005. Blood Flow and Permeability in Microvessels, Fluid Dynamics Research, 37, 82-132.

Suresh, S., Spatz, J., Mills, J. P., Micoulet, A., Dao, M., Lim, C. T., Beil, M., Seufferlein, T., 2005. Connections between Single-Cell Biomechanics and Human Disease States: Gastrointestinal Cancer and Malaria, Acta Biomaterialia, 1, 15-30. 


\section{Table and Figure captions}

Table 1: Dimensions of the sensor $(\mu \mathrm{m})$.

Table 2: Average values of the major and minor axes of the RBC and the deformation index, DI.

Table 3: Coefficient of variance for $\Delta R_{0}$ and $\delta$.

Figure 1: Schematic views of the proposed method of measuring method RBC deformability.

Figure 2: Equivalent circuit model of a RBC suspended in solution between the electrodes.

Figure 3: Schematic of the micro-channel and electrodes of the sensor.

Figure 4: Schematic of the impedance circuit diagram.

Figure 5: Spherical model of a RBC with cytoplasm and cytomembrane.

Figure 6: $\varepsilon_{\text {sus }}$ and $\kappa_{\text {sus }}$ of spherical RBCs suspended in PBS solution for $\phi=0.05$.

Figure 7: The effects of AC frequency on resistance of the solution.

Figure 8: Experimental apparatus.

Figure 9: Photo of the electrodes and forward-facing step.

Figure 10: Photos of a normal RBC passing between the electrodes.

Figure 11: Photos of a rigidified RBC passing between the electrodes.

Figure 12: Distribution of $\Delta R_{\mathrm{x}}$ against the streamwise position of RBC, $x_{\mathrm{RBC}}$.

Figure 13: Distribution of the maximum peak of the $\Delta R_{\mathrm{x}}$ profile, $\Delta R_{0}$, and half-width of the maximum of the $\Delta R_{\mathrm{x}} / \Delta R_{0}$ profile, $\delta$, against the spanwise position of RBC, $y_{\mathrm{RBC}}$.

Figure 14: Relation of $\Delta R_{0}$ and $\delta$ to deformation index, $D I$. 
Table 1: Dimensions of the sensor $(\mu \mathrm{m})$.

\begin{tabular}{ccccccc}
$W$ & $H_{1}$ & $H_{2}$ & $w_{\mathrm{s}}$ & $l_{\mathrm{s}}$ & $l_{\mathrm{sg}}$ & $l_{\mathrm{g}}$ \\
\hline 1000 & 52 & 10 & 5.2 & 14.8 & 2.3 & 14.6 \\
\hline
\end{tabular}


Table 2: Average values of the major and minor axes of the RBC and the deformation index, DI.

\begin{tabular}{lccccccc}
\hline \hline & samples & $a(\mu \mathrm{m})$ & SD of $a$ & $b(\mu \mathrm{m})$ & SD of $b$ & $D I$ & SD of $D I$ \\
\hline Normal RBC & 52 & 14.5 & 0.90 & 4.7 & 0.57 & 0.52 & 0.055 \\
\hline Rigidified RBC & 20 & 7.4 & 0.51 & 7.4 & 0.57 & 0 & 0.036 \\
\hline
\end{tabular}


Table 3: Standard variations for $\Delta R_{0}$ and $\delta$.

\begin{tabular}{ccc|cc}
\hline \hline \multicolumn{3}{c|}{$\Delta R_{0}$} & \multicolumn{2}{c}{$\delta$} \\
\hline Normal RBC & Rigidified RBC & Normal RBC & Rigidified RBC \\
\hline samples & 52 & 20 & 52 & 20 \\
\hline$\sigma$ & $14.2(\Omega)$ & $19.6(\Omega)$ & $0.84(\mu \mathrm{m})$ & $1.54(\mu \mathrm{m})$ \\
\hline$C v(\%)$ & 12.1 & 15.4 & 3.42 & 7.39 \\
\hline
\end{tabular}




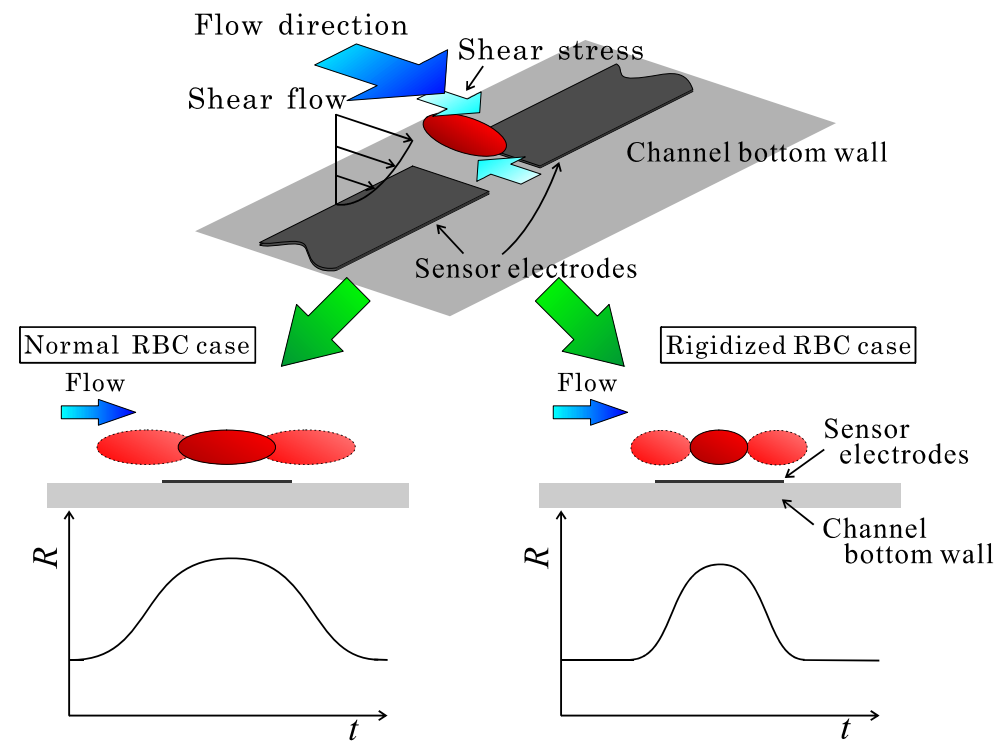

Figure 1: Schematic views of the proposed method of measuring method RBC deformability. 


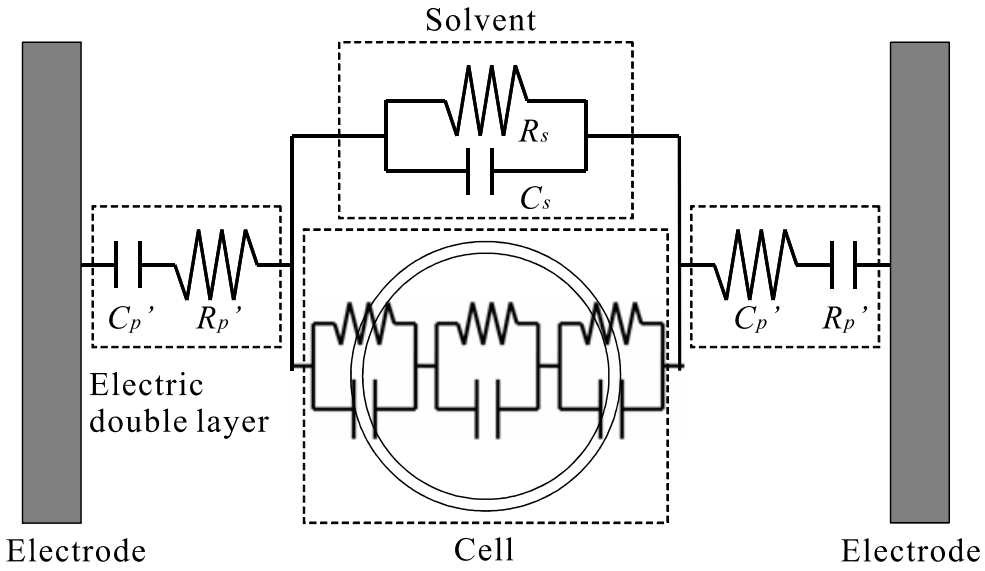

(a) Equivalent circuit

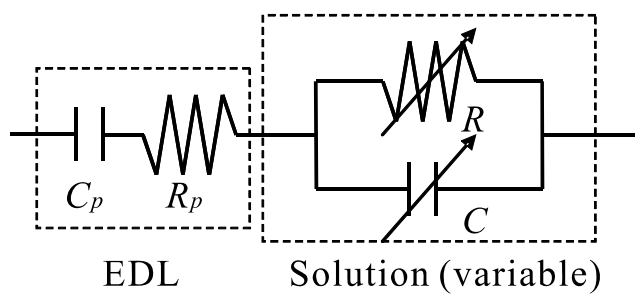

(b) Simplified ciruit model

Figure 2: Equivalent circuit model of a RBC suspended in solution between the electrodes. 


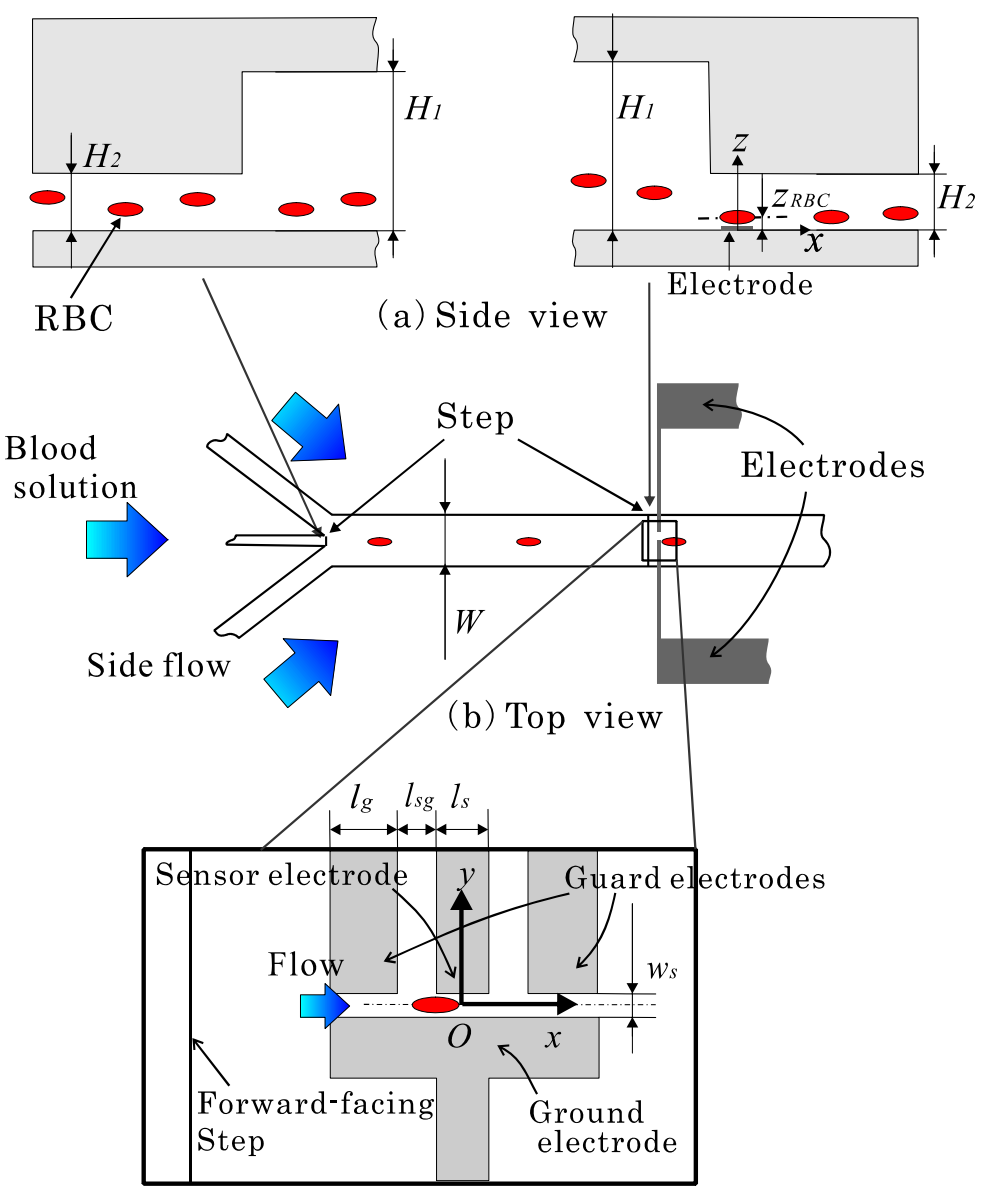

(c) Magnified top view of the electrodes

Figure 3: Schematic of the micro-channel and electrodes of the sensor. 


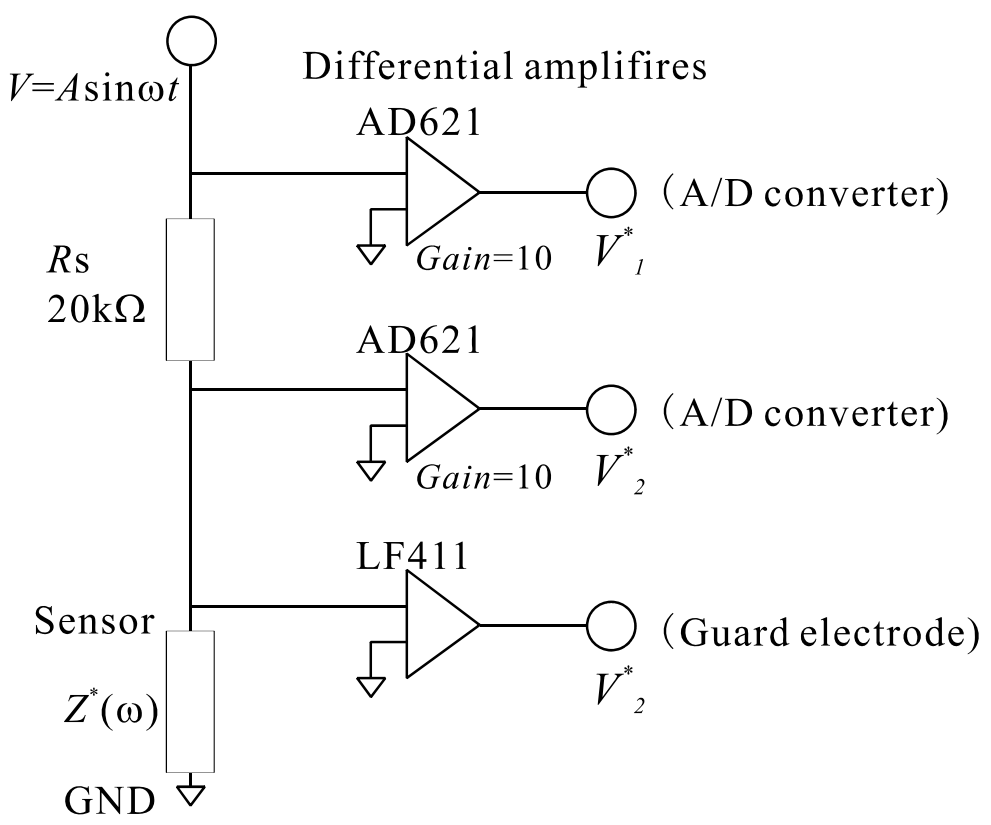

Figure 4: Schematic of impedance circuit diagram. 


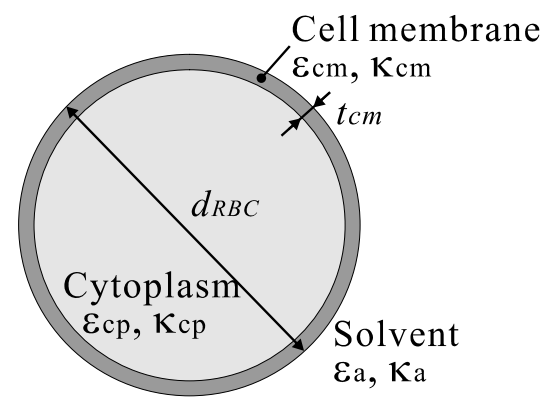

Figure 5: Spherical model of a RBC with cytoplasm and cytomembrane. 


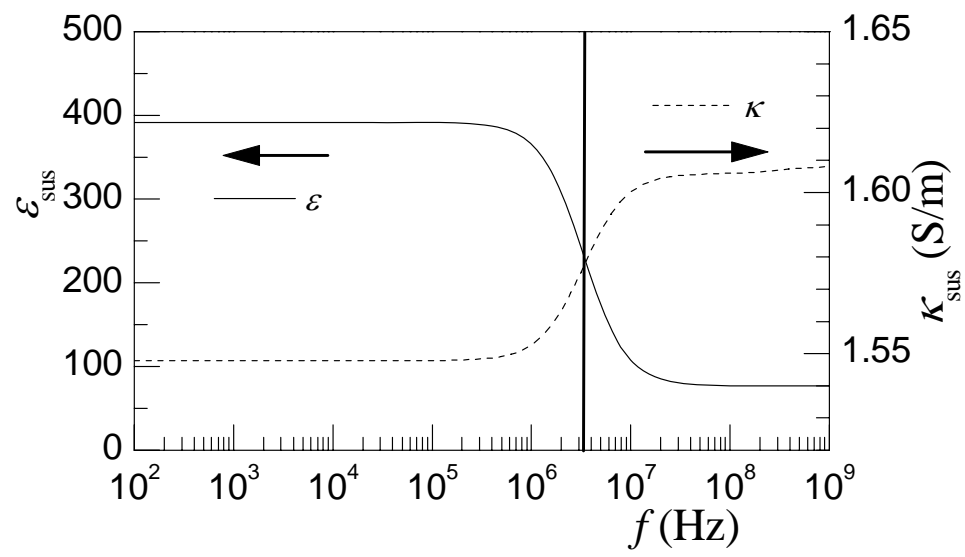

Figure 6: $\varepsilon_{\text {sus }}$ and $\kappa_{\text {sus }}$ of spherical RBCs suspended in PBS solution for $\phi=0.05$. 


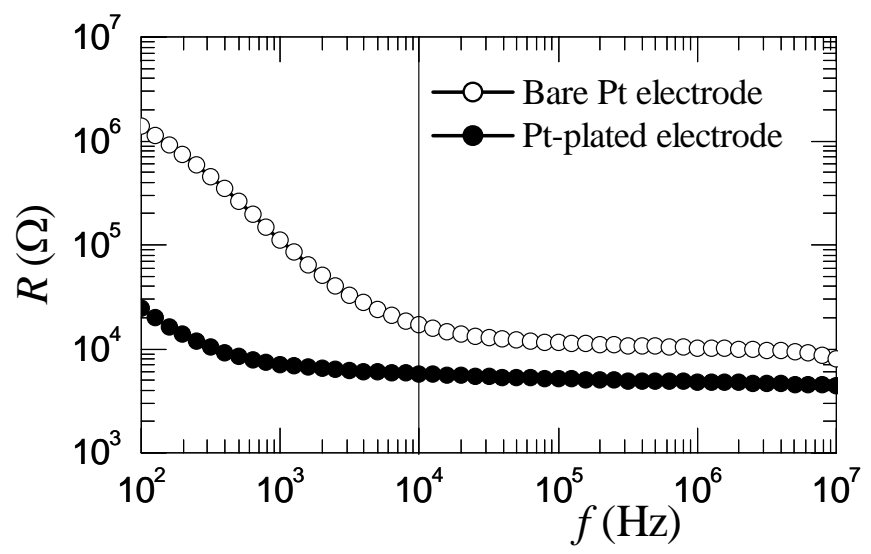

Figure 7: The effects of AC frequency on resistance of the solution. 


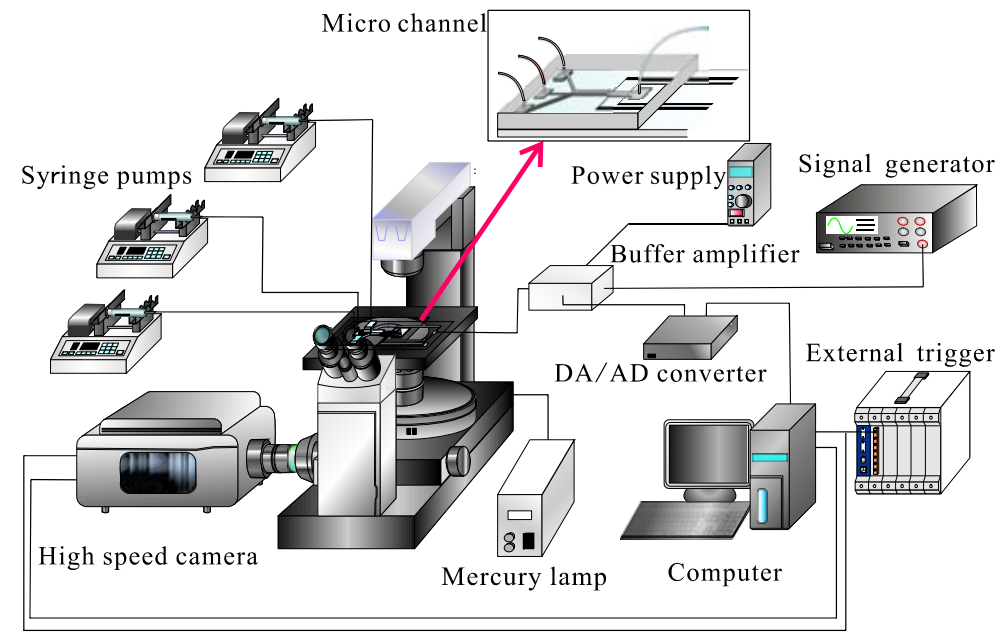

Figure 8: Experimental apparatus. 


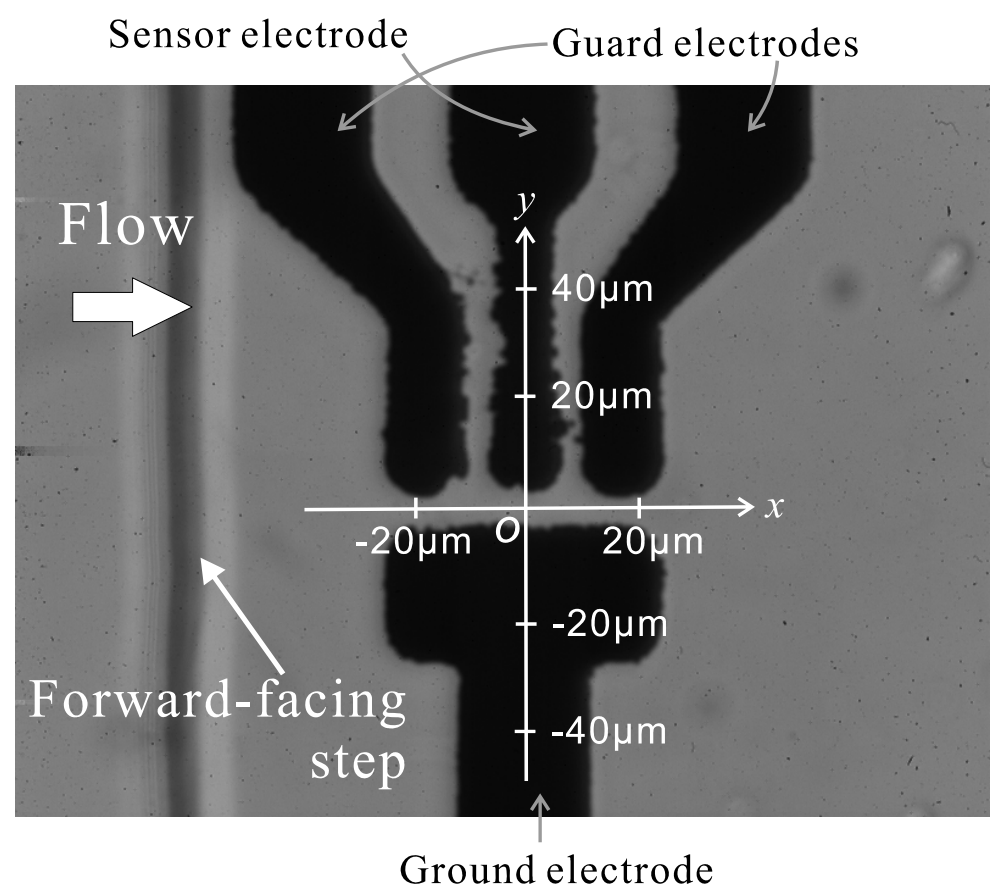

Figure 9: Photo of the electrodes and forward-facing step. 

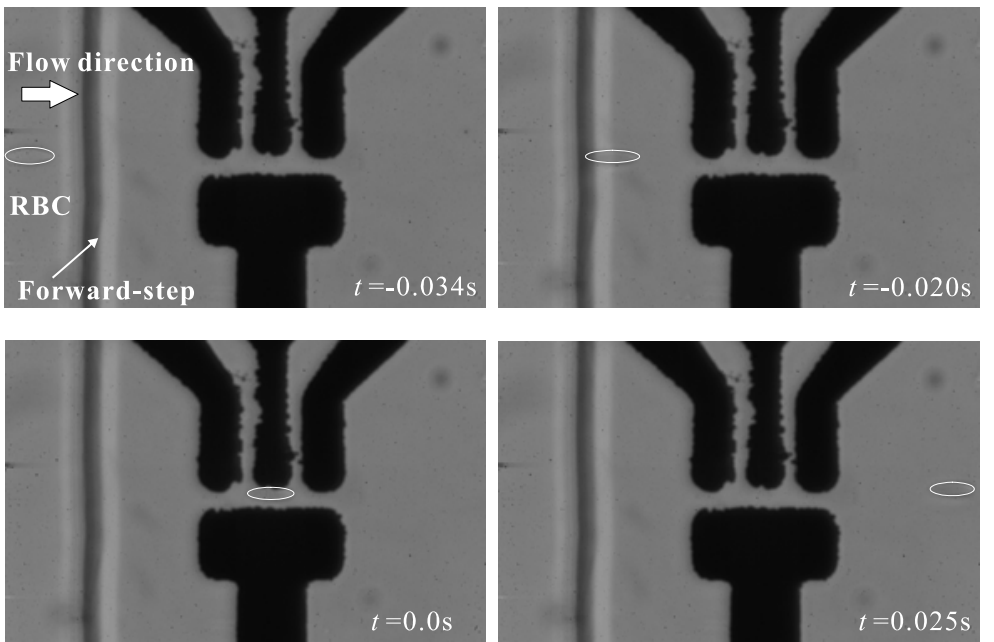

Figure 10: Photos of a normal RBC passing between the electrodes. 

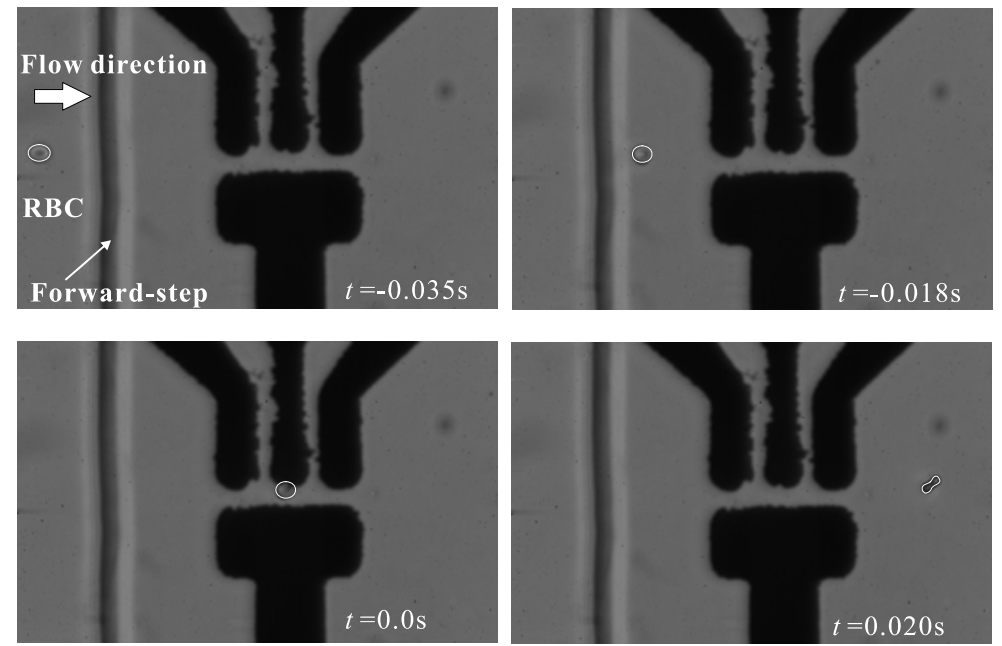

Figure 11: Photos of a rigidified RBC passing between the electrodes. 


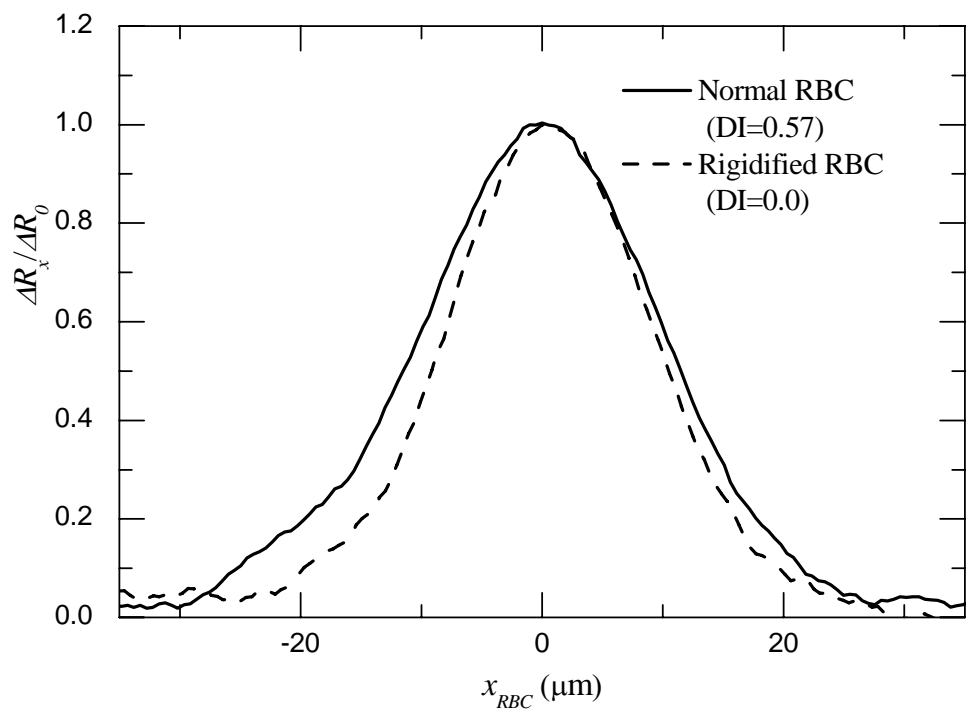

Figure 12: Distribution of $\Delta R_{\mathrm{x}}$ against the streamwise position of RBC, $x_{\mathrm{RBC}}$. 


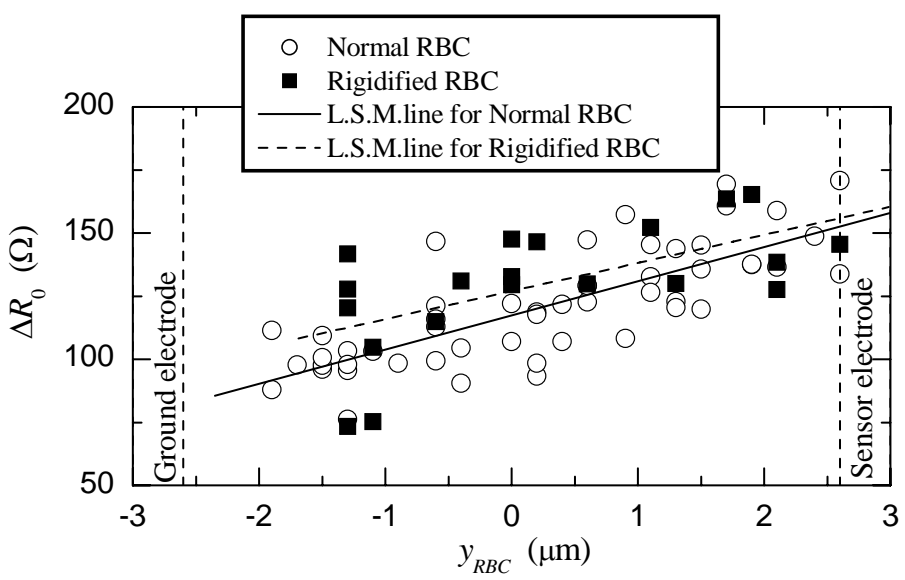

(a) $\Delta R_{0}$ vs $y_{\mathrm{RBC}}$

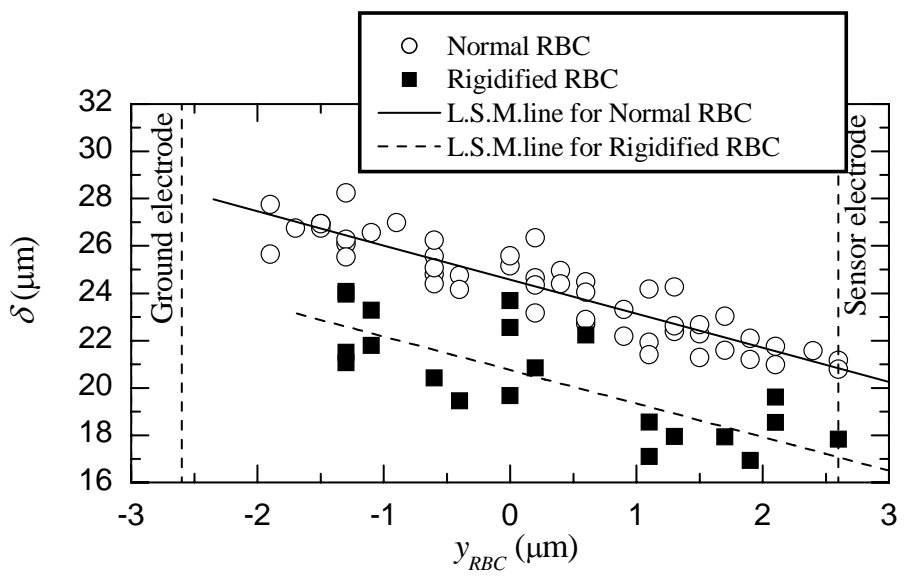

(b) $\delta \mathrm{vs} y_{\mathrm{RBC}}$

Figure 13: Distribution of the maximum peak of the $\Delta R_{\mathrm{x}}$ profile, $\Delta R_{0}$, and half-width of the maximum of the $\Delta R_{\mathrm{x}} / \Delta R_{0}$ profile, $\delta$, against the spanwise position of RBC, $y_{\mathrm{RBC}}$. 


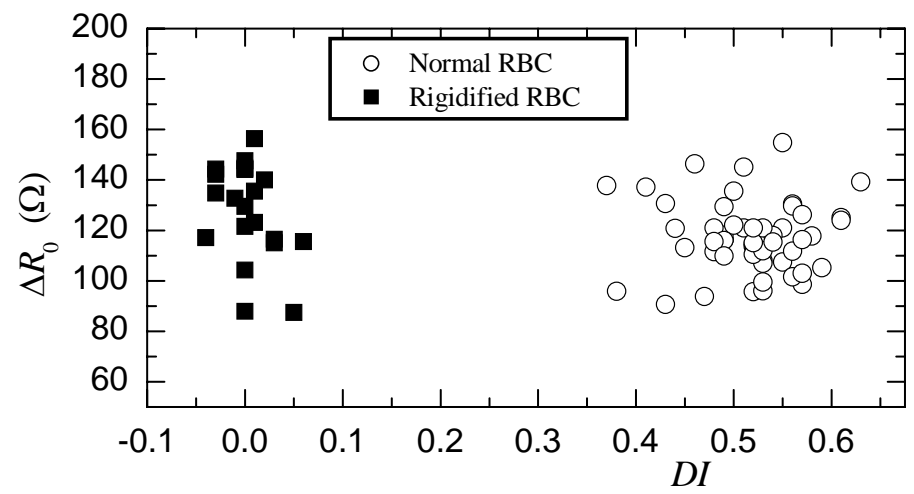

(a) $\Delta R_{0}$ vs $D I$

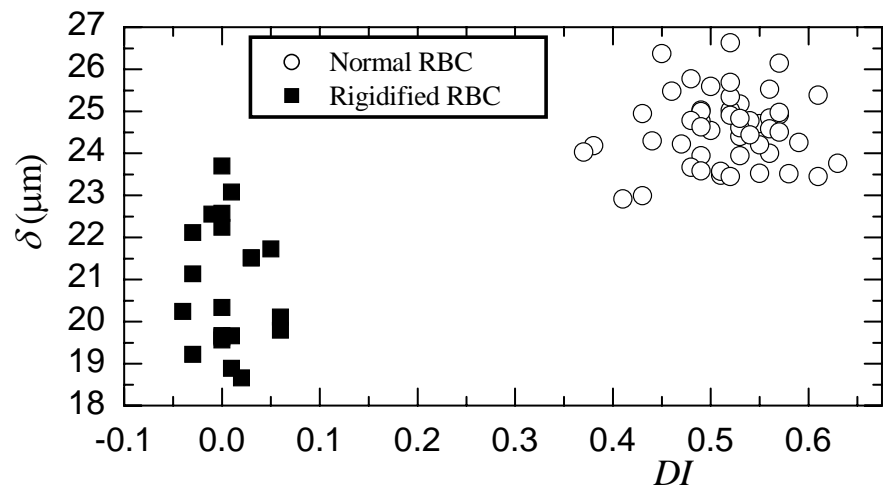

(b) $\delta$ vs $D I$

Figure 14: Relation of $\Delta R_{0}$ and $\delta$ to deformation index, $D I$. 